\title{
Memotine Hydrochloride
}

National Cancer Institute

\section{Source}

National Cancer Institute. Memotine Hydrochloride. NCI Thesaurus. Code C87665.

A hydrochloride salt form of memotine, an isoquinoline with activity ag ainst myxoviruses and paramyxoviruses. 